\title{
ON THE REFLECTION OF SOLITONS OF THE CUBIC NONLINEAR SCHRÖDINGER EQUATION
}

\author{
THEODOROS KATSAOUNIS AND DIMITRIOS MITSOTAKIS
}

\begin{abstract}
In this paper we perform a numerical study on the interesting phenomenon of soliton reflection of solid walls. We consider the $2 \mathrm{D}$ cubic nonlinear Schrödinger equation as the underlying mathematical model and we use an implicit-explicit type Crank-Nicolson finite element scheme for its numerical solution. After verifying the perfect reflection of the solitons on a vertical wall, we present the imperfect reflection of a dark soliton on a diagonal wall.
\end{abstract}

\section{IntRODUCTION}

In this paper we study numerically the phenomenon of reflection of bright and dark solitons of walls. To this effect, we consider the initial value problem of the cubic nonlinear Schrödinger (CNLS) equation

$$
\begin{cases}\mathrm{i} u_{t}+\Delta u+\lambda|u|^{2} u=0 & \text { in } \Omega \times(0, T], \\ u(\cdot, 0)=u_{0} & \text { in } \Omega,\end{cases}
$$

where we assume that $\Omega \subset \mathbb{R}^{2}$, is a bounded, convex, polygonal domain, $T<\infty, \lambda \in \mathbb{R}$. For $\lambda \leq 0$, problem (1) is known as the defocusing cubic nonlinear Schrödinger equation, while If $\lambda>0$, problem (1) is called the focusing cubic nonlinear Schrödinger equation. The CNLS equation (1) is used as a mathematical model in various applications such as nonlinear optics and lasers, water waves, quantum hydrodynamics and Bose-Einstein condensates, [14]. The nature of soliton reflection to walls requires that (1) is augmented with boundary conditions. In our study we consider three type of boundary conditions, a) zero Dirichlet (2) or b) zero Neumann (3) condition, in the whole boundary $\partial \Omega$ of the domain, or c) zero Dirichlet and zero Neumann on disjoint parts of the boundary (4),

$$
\begin{aligned}
u & =0 \text { on } \partial \Omega \\
\frac{\partial u}{\partial n} & =0 \text { on } \partial \Omega \\
u & =0 \text { on } \partial \Omega^{D} \text { and } \frac{\partial u}{\partial n}=0 \text { on } \partial \Omega^{N}, \quad \partial \Omega=\partial \Omega^{D} \cup \partial \Omega^{N}, \partial \Omega^{D} \cap \partial \Omega^{N}=\emptyset,
\end{aligned}
$$

A standard calculation, c.f. [6], shows that the initial value problem (1), augmented with any of the aforementioned boundary conditions (2), (3) or (4), conserves two physical quantities: mass $M(\cdot)$ and energy $E(\cdot)$. In particular, we have

$$
M(t):=\|u(t)\|^{2}, \quad E(t):=\frac{1}{2}\|\nabla u(t)\|^{2}-\frac{\lambda}{4}\|u(t)\|_{L^{4}}^{4}, \quad \text { then } \quad M(t)=M(0), \quad \text { and } \quad E(t)=E(0) \quad t \geq 0,
$$

where $\|\cdot\|,\|\cdot\|_{L^{q}}$ denote the $L^{2}$ and $L^{q}$-norms in $\Omega$, respectively.

In this paper we focus on two aspects: a) we evaluate a Crank-Nicolson relaxation method for 2D domains discretized by completely unstructured grids and b) we study various reflections of solitons on walls for CNLS type (1) of equations either focusing or defocusing.

Nonlinear Schrödinger type equations can describe waves in optical fibers, [2], as well as rogue waves in the ocean, cf. e.g. [11]. In both cases the study of the interaction of the waves with structures imposes the study of the reflection of solitons on walls. On the other hand, it is well known that in one space dimension CNLS equation (1) is an integrable system, thus one can compute analytically exact solutions describing soliton reflections. However, in two space dimensions system (1) is not integrable thus one has to rely on numerical methods for computing and studying such reflective phenomena.

The numerical method used here is based on the standard finite element method for the spatial discretization and the relaxation Crank-Nicolson scheme as a time marching mechanism. The relaxation Crank-Nicolson was introduced by Besse [3] can be viewed as a linearization method for the CNLS and as such it avoids the computationally expensive solution of a nonlinear equation at each time step

Key words and phrases. nonlinear Schrödinger equation, relaxation method, soliton reflection, dark and bright solitons. 
of the algorithm. Moreover, the relaxation scheme exhibits mass conservation, same like the standard Crank-Nicolson scheme, thus preserving the mass conservation property of the continuous problem, cf. (5). However, the relaxation scheme does not preserve the energy $E(\cdot)$ in two space dimensions. The relaxation Crank-Nicolson method is also used in [8] where optimal a posteriori error estimates for models of type (1) are obtained. Based on these a posteriori error estimates one can derive a space-time adaptive algorithm which will be able to capture all interested features of the solution with substantial reduction of the overall computational cost compared to that of uniform grids. A space-time adaptive algorithm based on a posteriori error estimates was developed in [7] for the linear Schrödinger equation. The numerical experiments reported in Section 3 are good examples where such a space-time adaptive algorithm will be very beneficial.

Due to the integrability properties of the CNLS equation, the reflection of a soliton can be studied analytically only in $1 \mathrm{D},[4,5,13]$. In this paper after verifying the order of accuracy of the numerical method in space and time, we validate the efficiency of the numerical method by studying first the perfect (elastic) reflection of dark and bright solitons on vertical walls using either (3) or (4) as boundary conditions, [4]. Finally, we show that the reflection of a dark soliton is not perfect (inelastic) when the soliton collides on the wall at an angle.

This paper is organized as follows: in Section 2 we describe briefly the relaxation Crank-Nicolson finite element method and state its approximation properties. Section 3 contains numerical results which a) validate the numerical method and b) study the phenomenon of soliton reflection.

\section{The numerical Method}

We describe briefly the numerical method used in this study. We start by presenting the relaxation Crank-Nicolson method and proceed by the fully discrete scheme. We consider a uniform partition $t_{n}=n k$ of $[0, T]$ where $k=T / N$ and $I_{n}:=\left(t_{n}, t_{n+1}\right], 0 \leq n \leq N-1$, denote the fixed time step and subintervals of $[0, T]$, respectively. For the spatial discretization, we consider a family of conforming, shape regular triangulations $\{\mathcal{T}\}$ of $\Omega$. For an element $K \in \mathcal{T}$, we denote by $h_{K}$ its diameter and let $h=\max _{K} h_{K}$. We also let $\mathbb{P}^{r}$ denotes the space of polynomials in two variables of degree at most $r$. Then to the triangulation $\mathcal{T}$ we associate a finite element space $\mathbb{V}_{r}$ which definition will depend upon the type of boundary condition we choose to work with. In particular we define

$$
\mathbb{V}_{r}:=\left\{\chi \in \mathbb{V}(\Omega): \forall K \in \mathcal{T},\left.\chi\right|_{K} \in \mathbb{P}^{r}\right\},
$$

where $\mathbb{V}=H_{0}^{1}(\Omega)$ for homogeneous Dirichlet condition $(2), \mathbb{V}=H^{1}(\Omega)$ for the homogeneous Neumann boundary condition (3) and $\mathbb{V}=H_{0}^{1}\left(\Omega^{D}\right)$ in the case of the mixed type boundary condition (4).

We can define now the relaxation Crank-Nicolson-Galerkin-type fully discrete scheme. Let $\bar{\partial} U^{n}:=$ $\left(U^{n+1}-U^{n}\right) / k$ and $U^{n+\frac{1}{2}}:=\left(U^{n+1}+U^{n}\right) / 2$. Then we seek approximations $U^{n} \in \mathbb{V}_{r}$ to $u\left(t_{n}\right)$ such that, for $0 \leq n \leq N-1$,

$$
\left\{\begin{array}{l}
\left\langle\frac{1}{2}\left(\Phi^{n+\frac{1}{2}}+\Phi^{n-\frac{1}{2}}\right), \chi\right\rangle=\left\langle\left|U^{n}\right|^{2}, \chi\right\rangle, \quad \forall \chi \in \mathbb{V}_{r}, \\
\mathrm{i}\left\langle\bar{\partial} U^{n}, \chi\right\rangle-\left\langle\nabla U^{n+\frac{1}{2}}, \nabla \chi\right\rangle+\lambda\left\langle\Phi^{n+\frac{1}{2}} U^{n+\frac{1}{2}}, \chi\right\rangle=0, \quad \forall \chi \in \mathbb{V}_{r},
\end{array}\right.
$$

where $\langle\cdot, \cdot\rangle$ denotes the $L^{2}$ - inner product, $\Phi^{-\frac{1}{2}}=\mathcal{P}\left(\left|u_{0}\right|^{2}\right), U^{0}=\mathcal{P} u_{0}$ with $\mathcal{P}$ being the $L^{2}$-projection $\mathcal{P}: L^{2} \rightarrow \mathbb{V}_{r}$. Formally we expect the method to be second order accurate in time and of $r+1$-order accurate in space, which can be expressed by an error estimate of the following form

$$
\max _{0 \leq n \leq N}\left\|U^{n}-u\left(t_{n}\right)\right\| \leq C\left(h^{r+1}+k^{2}\right),
$$

where $C$ is a constant depending on the exact solution $u$ of (1) and data of the problem, but it is independent of $h$ and $k$.

At each time step $t_{n}$, given an approximation $U^{n} \in \mathbb{V}_{r}$ and $\Phi^{n-\frac{1}{2}}$, the algorithm proceeds first by computing the new value $\Phi^{n+\frac{1}{2}}$. The computational cost of this update is relatively low since it involves only the cost of the projection of the right hand side in the first relation of (6). The cost of such projection amounts to the solution of a real linear system with the mass matrix and real right hand side since $\Phi^{n+\frac{1}{2}}$ is real valued. The second part of the algorithm (6), involves the update of the right hand side via a projection and the solution of a complex-valued linear system. The system matrix of this linear system, does not change in time, thus can be computed and factored only once in the initial step of the algorithm. An alternative approach for solving the linear systems is by using an appropriate conjugate gradient type method, since the system matrices in both steps are positive definite. Therefore 
the algorithmic complexity of method (6) at each time step is at most of $O\left(m^{2}\right)$ where $m$ denotes the size of the matrix, thus making the method computationally very attractive.

\section{NumERICAL EXPERIMENTS}

In this section we first validate numerically the method (6) by means of verifying the formal convergence rates in space and time of the formal error estimate (7). Furthermore, we present some results concerning the head on or oblique soliton reflection of solid walls.

In all, expect one, of the numerical experiments, we used unstructured triangulations of Delaunay type produced by the Bowyer-Watson algorithm, [12]. Delaunay triangulations have several advantages compared to uniform triangulations with the first being that are better suited for general geometries of the domain. Further, one of characteristics of the Delaunay triangulations is that maximizes the minimum interior angle of the triangles and on the same time minimize the maximum interior angle of the triangles, which guarantees triangles of good quality. This characteristic is preserved even when the triangulation is refined and/or coarsened locally and the resulting triangulation is also Delaunay. However this is not the case with uniform triangulations since local refinement and/or coarsening can deteriorate the quality of triangles rather fast and produce triangles which are elongated and "thin" with very small angles. Uniform triangulations may favour specific geometric directions in the domain which can influence the computed solution; such an issue is not present in an unstructured Delaunay triangulation.

3.1. Method validation. To validate the method numerically we perform a series of numerical experiments verifying the order of convergence as presented by the formal a priori error estimate (7). To facilitate the process and be able to compute the exact error between the true and approximate solution, we choose to work with a solution of a non-homogeneous version of equation (1) and in particular we choose

$$
u(x, y, t)=e^{t} x(1-x) y(0.5-y),
$$

which is an exact solution of (1) with appropriate right-hand side, with $\lambda=-2$, zero Dirichlet boundary conditions $(2)$, and $u_{0}(x, y)=u(x, t, 0)$. For the computation of the errors in time we use quadratic finite elements $(r=2)$ in space, the computational domain is the rectangle $\Omega=[0,1] \times[0,0.5]$ and final time $T=4$. The domain is covered by an unstructured in general triangulation $\mathcal{T}$ of good quality. To verify the error convergence rates we compute the experimental order of convergence (EOC). Let $\ell \in \mathbb{N}$ count the different realizations(runs) and let $h_{\ell}, k_{\ell}, \mathcal{E}_{\ell}$ be the spatial mesh size, time step and error respectively. Choosing a very small spatial mesh size, we used an unstructured triangulation consisting of 192802 triangles, the corresponding spatial component of the error is negligible and the temporal EOC is computed as $\mathrm{EOC}=\frac{\log \left(\mathcal{E}_{\ell+1} / \mathcal{E}_{\ell}\right)}{\log \left(k_{\ell+1} / k_{\ell}\right)}$. The temporal EOC is found to be 2 and is presented Table 1 .

\begin{tabular}{cccccc}
\hline$\ell$ & 1 & 2 & 3 & 4 & 5 \\
\hline$k_{\ell}$ & 0.5 & 0.25 & 0.08 & 0.0625 & 0.03125 \\
$E_{\ell}$ & $9.02 \times 10^{-3}$ & $2.27 \times 10^{-3}$ & $2.35 \times 10^{-4}$ & $1.43 \times 10^{-4}$ & $3.49 \times 10^{-5}$ \\
EOC & - & 1.9880 & 1.9905 & 2.0020 & 2.0412 \\
\hline
\end{tabular}

TABLE 1. Temporal numerical errors and experimental orders of convergence

The spatial errors can be computed in a similar manner. Specifically, in order to estimate the spatial convergence rates we take the domain to be $\Omega=[0,2] \times[0,2]$ and exact solution $u(x, y, t)=e^{t}(1-$ $\cos (2 \pi x)) \sin (2 \pi y)$, with $u=0$ on the boundary, along with the appropriate non-homogeneous term. Now we take $T=0.1$ and $k=2 \cdot 10^{-5}$, thus the temporal component of the error is negligible. The triangulation now is structured(uniform) and consisted of equal right-angle triangles with perpendicular sides of length $h=\sqrt{2 / N}$, where $N$ is the number of triangles. The number of triangles we tested were $N=32,128,512,2048,8192,32768$. The numerical experiments confirmed the expected orders of convergence. The numerical results are depicted in Figure 1, where the results are presented in logarithmic scales. 


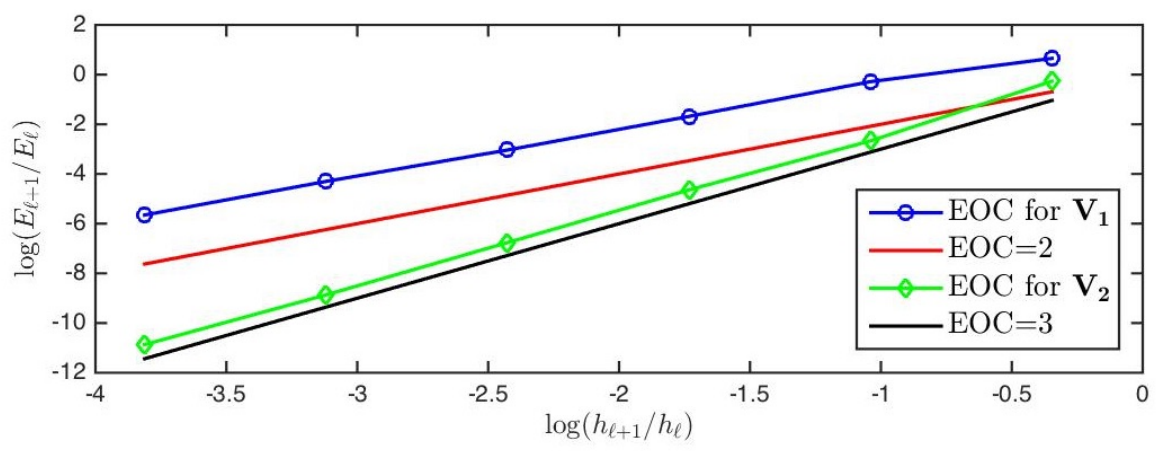

FiguRE 1. Experimental orders of convergence for linear and quadratic elements.

3.2. Perfect Reflection of Solitons. The focusing CNLS equation $(\lambda=2)$ has bright soliton solutions of the form $u_{b}(x, y, t)=\eta \operatorname{sech}[\eta(x+2 \xi t)] e^{-i \theta}$ where $\theta=\xi x+\left(\xi^{2}-\eta^{2}\right) t$, while the defocusing CNLS equation $(\lambda=-2)$ admits dark soliton solutions of the form $u_{d}(x, y, t)=\eta[\cos \xi+\mathrm{i} \sin \xi \tanh (\sin \xi \eta(-x+2 \eta \cos \xi t))] e^{-2 i \eta^{2} t}$, cf. e.g. [1]. The parameters $\xi$ and $\eta$ are chosen appropriately.

In this section we verify that the reflection of a soliton of the CNLS equation is perfect when the soliton collides with a vertical wall at zero angle. The reflection is called perfect if the reflected wave has the same shape as the original soliton but different direction of propagation. This behaviour has been studied analytically in $[4,5,13]$ for the integrable CNLS equation in one space dimension. We performed two numerical tests using boundary conditions (3) and (4). Both type of boundary conditions will give perfect reflections but the interaction of the soliton with the boundary in different. For the differences between the two reflections we refer to $[4,5]$.

In the first test we study the reflection of a bright soliton for the focusing CNLS equation with $\lambda=2, \eta=2, \xi=2$ and zero Neumann boundary conditions (3) in the domain $\Omega=[-5,5] \times[-1,1]$, while quadratic elements were used. The results of the perfect reflection are presented in Figure 2a, depicting the amplitude of the wave. It is known that the solitons of the CNLS equation suffer by an instability of focusing type. In order to ensure that the propagation of the soliton remains stable during the simulation we took 74496 triangles ensuring a very fine spatial grid and a small time-step $k=5 \times 10^{-3}$. During the experiment the mass $M$ was conserved with value 7.9999999 while the energy $E$ was conserved to 10.5 up to $T=3$.

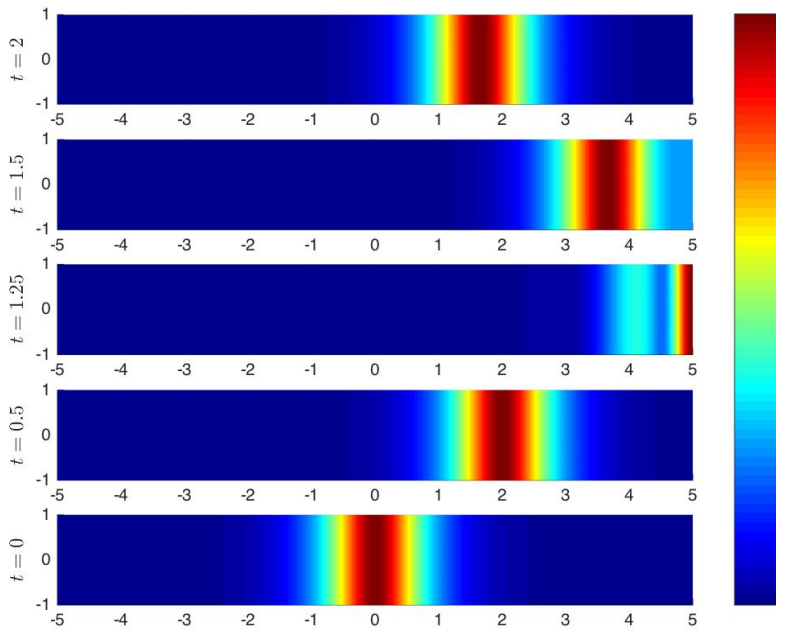

(A) Homogeneous Neumann boundary conditions (3).

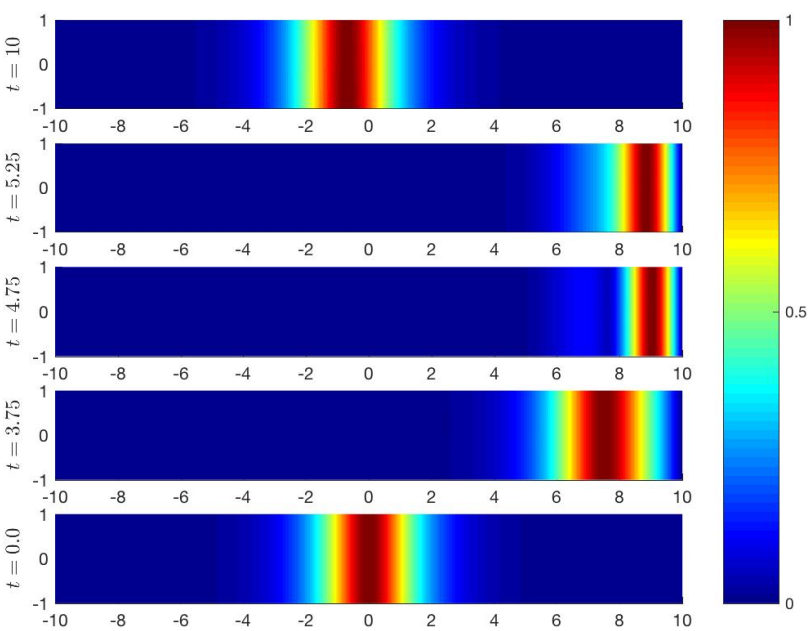

(B) Mixed boundary conditions (4).

Figure 2. Perfect reflection of bright solitons 
Our second test concerns also the reflection of a bright soliton for the focusing CNLS but in the case where the mixed boundary conditions (4) are used, c.f. $[4,5]$. In particular we took $\lambda=2, \eta=1, \xi=1$ in the domain $\Omega=[-10,10] \times[-1,1]$ and the zero Dirichlet conditions were applied at $x=-10$ and $x=10$ while homogeneous Neumann boundary conditions in the rest of the boundary of the domain. We have used a unstructured triangulation consisted of 74241 triangles, a time-step $k=2.5 \times 10^{-3}$ and quadratic finite elements. The results of the perfect reflection are presented in Figure $2 \mathrm{~b}$, depicting the amplitude of the wave. During the experiment the mass $M$ was conserved with value 3.9999999 while the energy $E$ was conserved to 1.3333 up to $T=10$.

Similarly, the perfect reflection of the dark soliton of the defocusing CNLS equation with $\lambda=-2$, $\eta=1, \xi=\pi / 4$ and with zero Neumann boundary conditions are presented in Figure 3 , showing the amplitude of the wave. Since the dark solitons are in general stable waves we used coarser grids than the previous experiment. Specifically, we used an unstructured mesh consisted of 18624 triangles and time-step $k=5 \times 10^{-2}$. Due to the stability properties of the defocusing CNLS equation, the mass $M$ was conserved with more digits than in the case of the focusing CNLS equation with value 17.1763733098 while the energy $E$ was conserved to 8.119 up to $T=15$.

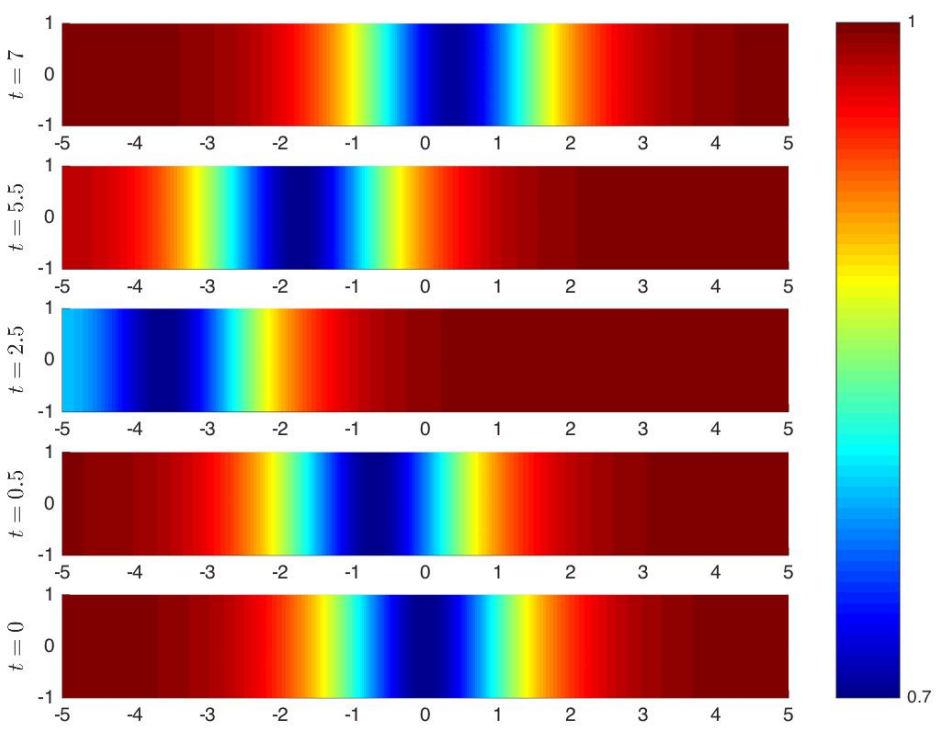

FiguRE 3. Perfect reflection of a dark soliton.

3.3. Reflection of a dark soliton on a diagonal wall. The reflection of the dark soliton on a vertical wall presented in the previous section was perfect in the sense that the reflected wave had the same shape with the original soliton. We close this paper with the study of the reflection of the same dark soliton of the defocusing CNLS on a diagonal wall. Since the CNLS equation in a two-dimensional domain is not integrable there is no analytical solution describing such a complicated reflection. The domain that we used here is a trapezoidal domain with vertices $(-8,-1),(-7,1),(8,1)$ and $(8,-1)$ while the triangular grid consisted of 28592 triangles. In this experiment we use also quadratic finite elements. The reflection is not perfect and is presented in Figure 4, depicting the amplitude of the wave. As the wave approaches the left side of the boundary, diffraction of the incident wave is being observed. The diffraction of the wave causes the distortion of the soliton while the reflected wave has an oscillatory structure in front and behind of the main pulse. Although it is known that dark solitons exhibit a transverse instability to perturbations with sufficiently long wavelenght, $[9,10]$, the reflected wave remained stable and no collapse or any other blow-up phenomenon was observed up to time $T=15$. During this experiment the mass $M$ retained the value (conserving the digits shown) 28.1716833830 and the energy $E$ was conserved to 13.61 up to $T=15$. Analogous observations can be made for the focusing CNLS equation but are not presented here. 


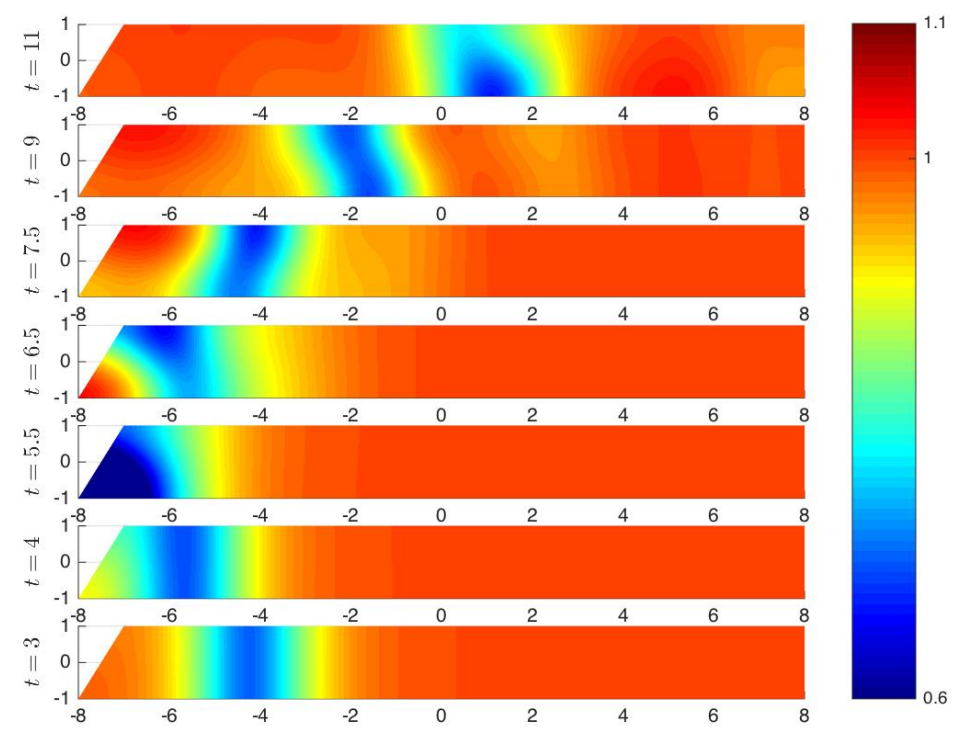

FIGURE 4. Reflection of a dark soliton by a diagonal wall.

Acknowledgements Dimitrios Mitsotakis would like to thank Profs G. Biondini, S. Flach and B. Ilan for valuable discussions on the properties of the CNLS equation. This work was supported by the Victoria University of Wellington Research Establishment Grant (Grand ID 208964). The authors would like to thank the anonymous referees for their valuable comments and suggestions.

\section{REFERENCES}

[1] M. Ablowitz, Nonlinear Dispersive Waves: Asymptotic Analysis and Solitons, Cambridge Texts in Applied Mathematics, 2011.

[2] G. Argawal, Nonlinear Fiber Optics, Academic Press, San Diego, 1989.

[3] Ch. Besse, A relaxation scheme for the nonlinear Schrödinger equation, SIAM J. Numer. Anal. 42 (2004) $934-952$.

[4] G. Biondini, G. Hwanng, Solitons, boundary value problems and a nonlinear method of images, J. Phys. A: Math. Theor. 42 (2009), 205207.

[5] G. Biondini, A. Bui, On the nonlinear Schrödinger equation on the half line with homogeneous Robin boundary conditions, Stud. Appl. Math. 129 (2012), 249-271.

[6] T. Cazenave, Semilinear Schrdinger equations, Courant Lecture Notes in Mathematics, 10. New York University, Courant Institute of Mathematical Sciences, American Mathematical Society, 2003

[7] Th. Katsaounis, I. Kyza, An adaptive finite element method for the linear Schrödinger equation, Numerische Mathematik, 129(2015), 55-90.

[8] Th. Katsaounis, I. Kyza, A posteriori error analysis for evolution nonlinear Schrödinger equations up to the critical exponent,arxiv:1601.02430v1.

[9] E.A. Kuznetsov, S.K. Tyritsyn, Instability and collapse of solitons in media with a defocusing nonlinearity, Sov. Phys. JETP 67 (1988), 1583-1586.

[10] M. Hoefer, B. Ilan, Dark solitons, dispersive shock waves, and transverse instabilities, Multiscale Model. Simul. 10 (2012), 306-341.

[11] A.R. Osborne, M. Onorato, M. Serio, The nonlinear dynamics of rogue waves and holes in deep-water gravity wave trains, Physics Letters A 275 (2000), 386-393.

[12] J. Shewchuk, Delaunay refinement algorithms for triangular mesh generation, Computational Geometry: Theory and Applications 22, (2002), 21-74.

[13] V. O. Tarasov, The integrable initial-boundary value problem on a semiline: nonlinear Schrödinger equation and sine-Gordons, Inv. Prob. 7 (1991), 435-449.

[14] C. Sulem, P.-L. Sulem, The nonlinear Schrödinger equation: self-focusing and wave collapse, vol. 139, Springer, 1999.

(Theodoros Katsaounis) King Abdullah University of Science and Technology(KAUST), Thumal, Kingdom of Saudi Arabia, \& IACM-Forth, Heraklion, Greece, \& Dept. of Math \& Appl. Mathematics, Univ. of Crete, GREECE

E-mail address: theodoros.katsaounis@kaust.edu.sa

(Dimitrios Mitsotakis) Victoria University of Wellington, School of Mathematics and Statistics, New Zealand,

\& IACM-Forth, Heraklion, Greece, \& Dept. of Math \& Appl. Mathematics, Univ. of Crete, Greece

E-mail address: dimitrios.mitsotakis@vuw.ac.nz 